\title{
Curriculumentwicklung im Zeitalter der Digitalisierung: Rahmenbedingungen, Herausforderungen, Formate und Inhalte
}

\section{Tobias Seidl und Antje Michel}

\section{Zusammenfassung}

Der mit der Digitalisierung verbundene gesellschaftliche Transformationsprozess wirkt sich auch auf die Gestaltung von Studiengängen aus. Basierend auf Erkenntnissen der Ad-Hoc-AG „Curriculum 4.0“ des Hochschulforums Digitalisierung sowie ihrer Curriculumentwicklungsprojekte im Rahmen der Förderlinie „Curriculum 4.0“ des Stifterverbands für die Deutsche Wissenschaft diskutieren die Autorin und der Autor dieses Beitrags drei Herausforderungen für die Weiterentwicklung von Curricula: den Stellenwert der Digitalisierung als kompetenzorientierter Lerninhalt (2), die Flexibilisierung von Curricula innerhalb von Akkreditierungszyklen zur Integration von digitalkompetenzorientierten Lernzielen und Lehr-Lern-Formen (3) sowie die digitale Kompetenzentwicklung der Lehrenden (4). Der Beitrag bietet einen fachlichen Überblick sowie Reflexionsimpulse für die praktische (Weiter-)Entwicklung von Studiengängen.

\section{Schlüsselwörter}

Curriculumentwicklung $\bullet 21$ st Century Skills • Digitale Lehre • Hochschullehre $\bullet$ Digitalisierung $\bullet$ Kompetenzziele $\bullet$ Lerninhalte $\bullet$ Lehr-Lern-Formate $\bullet$ Flexibilisierung

\section{T. Seidl $(\varangle)$}

Hochschule der Medien Stuttgart, Stuttgart, Deutschland E-Mail: seidl@hdm-stuttgart.de

A. Michel

Fachhochschule Potsdam, Potsdam, Deutschland

E-Mail: antje.michel@fh-potsdam.de 


\section{Digitale Transformation im Fokus der Curriculumentwicklung}

Digitalisierung und Globalisierung haben einen gesellschaftlichen Transformationsprozess ausgelöst, der vielfach disruptiv auf die Techniken und Praktiken des analogen Zeitalters wirkt. Zahlreiche Berufsbilder verändern sich massiv. Der Wandel betrifft Ideengenerierungs- und Produktionsweisen, Kommunikationspraktiken, institutionelle Organisationsformen, Geschäftsmodelle, Wertschöpfungsformen und soziale Rahmenbedingungen. Auch Prozesse der zivilgesellschaftlichen Teilhabe verändern sich, vor allem durch sich wandelnde Gesellschaftskonzepte, durch digitale, überregional bis teilweise global vernetzte Informations- und Kommunikationsmöglichkeiten und den Strukturwandel der Medien. Dieser Wandel führt auch zu der Ausbildung einer digital-analog integrierten Infosphäre; eine permanente Onlife-Lebensrealität prägt unsere menschliche Existenz. Der Informationsphilosoph Luciano Floridi, der diese Begriffe geprägt hat, bewertet die digitale Transformation deshalb als vierte Revolution (Floridi 2014).

Diese hier nur skizzierten massiven Veränderungsprozesse wirken auf das Selbstverständnis sowie die Gestaltung von Hochschule und Hochschullehre. Dies betrifft nicht nur die Entwicklung neuer Lehr-Lern-Formen und die Integration neuer Lerninhalte, sondern auch die grundsätzlichen Konzepte von Studium und Hochschule. Immer wieder wird in der bildungspolitischen Diskussion der Abgesang der Hochschule getätigt, wie zum Beispiel um die Jahrtausendwende mit dem Aufkommen der Massive Open Online Courses (MOOCs) und ihrer (vorschnellen) Beurteilung als disruptive Entwicklung für die Hochschullandschaft (Langen und Bosch 2014) und auch derzeit wieder als Wirkung des pandemiebedingten Shutdowns der Hochschule als Präsenzort (vgl. zum Beispiel Fuchs 2020).

Besonders deutlich wird der Transformationsprozess an einem der Kernprozesse der Hochschullehre: der (Weiter-)Entwicklung von Studiengängen und deren Curricula. Studiengangsentwicklung ist ein komplexer Prozess, in dem zunächst ein Studiengang fachlich konzipiert und dann sein Curriculum entwickelt wird. Schaper (2012) gliedert das Vorgehen in acht aufeinander aufbauende Schritte, dazu gehören etwa die Festlegung der Kompetenzziele, der zum Einsatz kommenden Lehr- und Lernformen sowie des Vorgehens bei der Evaluation des Lernerfolgs (vgl. Abb. 1).

Zwar existiert im Kontext von Bologna-Reform und Akkreditierungsrichtlinien eine Reihe hilfreicher Handreichungen zur Entwicklung von Studiengängen und Curricula; eine Auseinandersetzung mit der Frage, ob und, wenn ja, wie 
Umfeldanalyse

1. Vorklärung von Rahmenbedingungen und des Bedarfs für einen Studiengang;

Zieldefinition

2. Bestimmung des Qualifikations- bzw. Kompetenzprofils für einen Studiengang,

3. Beschreibung der Zielvorstellungen des Studiengangs sowie der zu erreichenden Lernergebnisse,

4. Bestimmung der fachlichen überfachlichen Kompetenzen, die mithilfe des Studiengangs erreicht werden sollen.

Curriculumgestaltung

5. Transformation der Zielvorstellungen und erwarteten Lernergebnisse in einen Lehrplan: Inhalt (abzudeckende Inhalte) und Struktur (Module und Credits),

6. Transformation in Lerneinheiten und Prüfungsleistungen, um die definierten Lernergebnisse zu erreichen,

7. Auswahl und Hinweise zu angemessenen Lehr-/Lernformen sowie Prüfungsformaten zur Umsetzung des Studiengangs.

Evaluation und Weiterentwicklung

8. Entwicklung eines Evaluationssystems mit dem Ziel, die Qualität des Studiengangs kontinuierlich zu verbessern.

Abb. 1 Vorgehen bei der Studiengangsentwicklung. (Eigene Darstellung nach: Schaper 2012, S. 54 ff.)

Digitalisierung und digitale Transformation als Faktoren in die Curriculumentwicklung einzubeziehen sind, fand jedoch bisher kaum statt. Die Förderlinie Curriculum 4.0 des Stifterverbands und der Carl-Zeiss-Stiftung (2017-2019) sowie die gleichnamige Ad-Hoc-AG des HFD (2017-2019) bündelten erste Pilotprojekte und Aktivitäten zu einer systematischen Auseinandersetzung mit dieser Thematik. So formulierte etwa die Ad-Hoc-AG eine Reihe von Empfehlungen sowohl zur Gestaltung von Freiräumen innerhalb bestehender Curricula als auch zu ihrer stakeholderorientierten Weiterentwicklung und der agilen Gestaltung von Curriculumentwicklungs- und Akkreditierungsprozessen (Hochschulforum Digitalisierung 2018).

Seit im Frühjahr 2020 die COVID-19-Pandemie den Präsenzbetrieb der Hochschullehre international betroffen und in vielen Regionen der Welt sprichwörtlich lahmgelegt hat, stellt sich nicht nur die Frage, ob die Möglichkeiten der Digitalisierung disruptives Potenzial für die Gestaltung unserer Präsenzlehre haben. Vielmehr können die Hochschulen in einer Art unfreiwilligem Experiment 
Erkenntnisse darüber generieren, wie krisenfest die Curricula bei derartigen disruptiven Ereignissen sind. Zudem erfahren gerade alle Hochschullehrenden in der täglichen Praxis, welchen Beitrag digitale Tools und Praktiken bieten, Hochschullehre auch in dieser Krisensituation zu realisieren, und wo die Grenzen hierfür sind. Es ist zur Zeit der Niederschrift dieses Beitrags noch zu früh, um aus diesen unsystematischen Beobachtungen systematische Lehren für die Gestaltung von Lehr-Lern-Formen sowie von Studiengängen zu ziehen, aber diverse Aktivitäten, die in diese Richtung zielen, formieren sich derzeit in der deutschen Hochschullandschaft. ${ }^{1}$ Eines zeichnet sich dabei ab: Die starke Formalisierung unserer Curricula ist ein Risiko für die flexible Reaktion auf Krisensituationen. Dies betrifft sowohl die geringe Flexibilität, Lehrinhalte über den Verlauf des akademischen Jahres neu anzuordnen und Lehr-Lern-Formen und die dazugehörigen klar definierten Prüfungen an die Krisensituation anzupassen, als auch die Möglichkeit, Inhalte, die sich spontan aus der Krisensituation ergeben, in die Curricula $\mathrm{zu}$ integrieren.

Im vorliegenden Beitrag werden aufbauend auf die bisherigen Arbeitsergebnisse der Ad-hoc-AG Curriculum 4.0 des HFD zwei maßgebliche Wechselwirkungen zwischen Digitalisierung und Studiengangsentwicklung in den Blick genommen:

- Zieldefinition: Den Stellenwert der Digitalisierung als Lerninhalt klären

- Curriculumgestaltung: Flexible und den Kompetenzzielen angepasste LehrLern-Formate, die von digital kompetenten Lehrenden angeboten werden

Die Auseinandersetzung mit den Wechselwirkungen zwischen Studiengangsentwicklung und Digitalisierung wird über kurz oder lang an allen Hochschulen stattfinden müssen. Auch wenn die zentralen Fragen an allen Hochschulen ähnlich sind, werden die Antworten abhängig von den Spezifika der einzelnen Standorte durchaus unterschiedlich ausfallen. Unser Beitrag will deshalb eine thematische Fundierung, aber auch praktische Impulse liefern, um die Diskussion an den Hochschulen anzuregen und zu lenken. Daher werden am Ende jedes Kapitels Leitfragen für die konkrete Praxis der Curriculumentwicklung vorgeschlagen.

\footnotetext{
${ }^{1}$ Vgl. exemplarisch \#Semesterhack des HFD (Hochschulforum Digitalisierung 2020) und Hochschul-Hackathon des Landes Brandenburg (Hochschul-Hackathon Brandenburg o. D.).
} 


\section{Zieldefinition}

\subsection{Kompetenzen für heute, morgen und übermorgen}

Ein Hochschulstudium zielt auf die Befähigung von Absolvent*innen zu einer wissenschaftlichen oder künstlerischen Tätigkeit, zur Aufnahme einer qualifizierten Berufstätigkeit, zur gesellschaftlichen Teilhabe sowie zur Selbstentfaltung (Akkreditierungsrat 2013). Diese übergeordneten Ziele der Hochschulbildung gelten für alle Hochschulen gleichermaßen, wenn auch mit unterschiedlichen Schwerpunktsetzungen. Bei der Studiengangsentwicklung müssen diese Ziele berücksichtigt, aber individuell austariert werden (Gerholz und Sloane 2013). Dabei ist die kritische Reflexion der Kompetenzziele, Inhalte und Lehr-LernMethoden im Hinblick auf die Auswirkungen eines gesellschaftlichen und technischen Wandels für alle Hochschulen von hoher Relevanz. Also betrifft der eingangs skizzierte Veränderungsprozess sowohl die Praxis der Hochschullehre insgesamt als auch die Qualifizierungsziele einzelner Curricula.

Für einen umfassenden Blick auf die Kompetenzen im Rahmen der Studiengangsentwicklung hilft es, die Kompetenzen gemäß ihrer Relevanz für heute, morgen und übermorgen zu differenzieren (vgl. dazu auch Seidl 2017):

1. Heute: Welche Kompetenzen benötigen Studierende, um ihr Studium erfolgreich absolvieren zu können?

2. Morgen: Welche Kompetenzen benötigen Studierende, um in der momentanen Lebens- und Arbeitswelt erfolgreich bestehen zu können?

3. Übermorgen: Welche Kompetenzen benötigen Studierende, um die Lebensund Arbeitswelt der Zukunft kompetent mitgestalten zu können?

Der Bedarf auf den Ebenen des Heute und Morgen läßt sich durch Einbeziehung von Stakeholdern (zum Beispiel durch die Beteiligung von Studierenden, Lehrenden, potenziellen Arbeitgeber*innen und Absolvent*innen) erheben. Zudem können Arbeits- und Aufgabenanalysen zur Definition notwendiger Kompetenzen herangezogen werden. Für einige Fächer liegen etwa berufsbezogene Qualifikationsrahmen vor, die zumeist in Kooperation von Fachgesellschaften, Hochschulen und Wirtschaftsvertreter*innen erstellt wurden. Die darin formulierten Kompetenzprofile können als Orientierung für die Studiengangsentwicklung dienen. Über diese traditionellen Vorgehensweisen hinaus lohnt es sich, bei der Stakeholderintegration auch neue methodische Ideen in den Blick zu nehmen, beispielsweise: 
- Studierende: Einbindung über Semesterprojekte zur Visionsentwicklung des eigenen Studien- und Berufsfelds (vgl. Michel et al. 2018) oder Durchführung von Student Days (Venn 2013)

- Alumni: Einbindung mit Interaktionsformen, die der Fachcommunity entsprechen (vgl. Michel et al. 2019)

- Externe Peers: Curriculumentwicklung zum Thema der Fachdiskussion machen (zum Beispiel Fachtagung/Workshop), Einbindung jenseits Gutachter*innen-Prozesse (vgl. Digitale-Lehre-Germanistik 2020)

Mit der Frage nach dem Übermorgen haben sich bereits diverse Studien - vorrangig aus der Perspektive der Employability - auseinandergesetzt (vgl. zum Beispiel World Economic Forum 2016). Die jüngste und auf Deutschland bezogene Studie wurde 2019 vom Stifterverband in Zusammenarbeit mit McKinsey vorgelegt. Mit einem Mixed-Methods-Ansatz wurden Trends und Bedarf der deutschen Wirtschaft erhoben, mit dem Ziel, Kompetenzlücken zu antizipieren und dadurch Impulse für Bildungspolitik und Bildungseinrichtungen zu geben. Im Ergebnis wird eine dreiteilige Kategorisierung der notwendigen Skills vorgeschlagen, mit der Unterscheidung von technologischen Fähigkeiten, wie zum Beispiel Webentwicklung oder Smart-Hardware-Entwicklung, digitalen Grundfähigkeiten, wie zum Beispiel digitale Lernkompetenz und digitale Informationskompetenz, und klassischen Fähigkeiten, wie zum Beispiel Adaptationsfähigkeit oder Kreativität (Kirchherr et al. 2019).

\subsection{Ein Kompetenzrahmen als Matrix für die Curriculumentwicklung}

Noch einen Schritt weiter geht das Konzept der 21st Century Skills. Unter diesem Stichwort haben sich weltweit verschiedene Expert*innenorganisationen der Herausforderung gestellt, zukünftig benötigte Kompetenzen näher zu definieren und zu operationalisieren. Der hier verwendete Begriff Skill, im Sinne von Fähigkeit, ist leider etwas verkürzend, da es in der Diskussion eigentlich um Kompetenzen im Sinne der Befähigung geht, ,in bestimmten Anforderungsbereichen angemessen, verantwortlich und erfolgreich zu handeln" (Schaper 2012, S. 93). Kompetenzen bestehen stets aus einem Zusammenspiel von Wissen, Fertigkeiten/Anwendungsfähigkeiten sowie motivationaler Orientierung und (Wert-)Haltung. Lernprozesse mit dem Ziel, Kompetenzen zu erwerben, finden also immer auf allen drei Ebenen statt. 
Orientiert an diesem ganzheitlichen Kompetenzverständnis hat eine Forscher*innengruppe die Inhalte verschiedener 21st-Century-Skills-Modelle zusammengefasst und konsolidiert. Das dabei entstandene KSAVE-Modell gleicht so die Limitationen einzelner Studien aus (Binkley et al. 2012). Das so entwickelte Metamodell besteht aus zehn Kompetenzbereichen, die jeweils auf den drei Ebenen (1) Knowledge, (2) Skills und (3) Attitudes, Values und Ethics beschrieben werden. Anders als in den vorher benannten Studien liegt der Fokus für die Identifikation der Kompetenzbereiche nicht allein auf der Berufsfähigkeit, sondern auf einer übergreifenden Perspektive der erfolgreichen Teilhabe an gesellschaftlicher Interaktion. Die zehn Kompetenzbereiche werden vier übergreifenden Kategorien zugeordnet.

Ways of Thinking

1. Kreativität und Innovation

2. Kritisches Denken, Problemlösung, Entscheidungsfähigkeit

3. Lernen lernen und Metakognition

Ways of Working

4. Kommunikation

5. Zusammen- und Teamarbeit

Tools for Working

6. Informationskompetenz

7. Umgang mit Informations- und Kommunikationstechnologien

Living in the World

8. Gesellschaftliches Engagement (lokal und global)

9. Leben- und Karriereplanung/-gestaltung

10. Eigenverantwortung und soziale Verantwortung (inklusive kultureller Sensibilität und interkultureller Kompetenz)

Das KSAVE-Modell hat für die Studiengangsentwicklung zwei entscheidende Vorteile: Zum einen kann es als abgesicherte Quelle für die Ableitung von notwendigen Zukunftskompetenzen für Beruf und Alltag genutzt werden. Zum 
anderen sind die Kompetenzen im Modell so weit operationalisiert und ausformuliert, dass sie einfach in Lern-/Kompetenzziele überführt werden können. Diese Operationalisierung erfolgt auf allen drei Ebenen von Kompetenz und ist damit in hohem Maße anschlussfähig an einen ganzheitlichen akademischwissenschaftlichen Kompetenzbegriff (Schaper 2012; Seidl et al. 2018).

Das KSAVE-Modell ist nicht explizit für die Gestaltung von Curricula für das digitale Zeitalter entwickelt worden. Dennoch bietet es hierfür den Schlüssel, indem je nach Studiengang und übergeordneten Bildungszielen die Kompetenzbereiche durch konkrete Lernziele hinterlegt werden können. Wenn die zu Beginn dieses Abschnitts beschriebenen normativen Ziele der Studiengangsentwicklung auf die digitale Transformation bezogen werden, zeigt sich, dass spezifische Herausforderungen und daraus resultierende Lernziele im Zusammenhang mit einer zunehmend digitalen Lebens- und Arbeitswelt je nach Studiengang aus fast jedem Kompetenzbereich des KSAVE-Modells ableitbar sind (vgl. zur Umsetzung zum Beispiel Burmester und Seidl 2020).

Methodisch können im Rahmen eines Studiengangsentwicklungsprozesses die Kompetenzen für das Übermorgen auf unterschiedliche Arten für den spezifischen Studiengang ausformuliert werden. Beispielsweise können Zukunftswerkstätten unter Beteiligung interner und externer Stakeholdergruppen durchgeführt werden, die sich mit der zukünftigen gesellschaftlichen Entwicklung und den erwarteten Veränderungen des Berufsfeldes auseinandersetzen (vgl. Element Zukunftswerkstatt im Konzept Curriculumswerkstatt der TH Köln [TH Köln o. D.]). Studierende können sehr gut über ein- oder zweisemestrige Zukunftsforschungsseminare zur Entwicklung von studentischen Visionen für die Weiterentwicklung ihrer Profession und daraus abzuleitender zukunftsorientierter Kompetenzen eingebunden werden (vgl. Michel et al. 2018). Eine weitere Möglichkeit ist der Einsatz einer Delphi-Studie, in der die Perspektiven externer Expert*innen gezielt herausgearbeitet werden können (vgl. HRK Projekt Nexus 2017).

Für die Curriculumentwicklungspraxis empfehlen wir, die Entwicklung zukunfts- und kompetenzorientierter Lernziele an folgenden Leitfragen zu orientieren:

- Welche Methoden können zur Identifikation von zukunftsorientierten Kompetenzen unter Einbindung von Stakeholdern der Studiengänge eingesetzt werden?

- Welche Kompetenzen brauchen unsere Absolvent*innen, um für die Zukunft gerüstet zu sein? Über welche verfügen sie bereits und welche sind bereits Teil des Curriculums? 
- Inwieweit herrscht Konsens in der Hochschule, dass die Ausbildung von (Wert-)Haltung oder Einstellungen Teil des Auftrags akademischer Lehre ist?

- Wie können wir in der Lehre einen sinnvollen Rahmen schaffen, damit diese Kompetenzen (bestehend aus Wissen, Fertigkeiten, Fähigkeiten sowie motivationaler Orientierung und [Wert-]Haltung) von den Studierenden erworben werden können?

\section{$3 \quad$ Flexible und agile Gestaltung von Curricula}

Die digitale Transformation erfordert, der oben dargestellten Argumentation folgend, in Bezug auf die zu entwickelnden Kompetenzen der Studierenden weniger ein spezifisches Skill-Set, sondern bildet vielmehr einen Bezugspunkt für die Entwicklung von Kompetenzbereichen und muss also bei der Formulierung von Kompetenzentwicklungs- und Lernzielen in jedem möglichen Bereich mitgedacht werden. Gemäß den Erkenntnissen aus den Expertiseworkshops der Ad-Hoc-AG des HFD beeinflussen insbesondere zwei Merkmale der globalisierten und zunehmend digitalisierten Gesellschaft den Prozess der Curriculumentwicklung, auf die reagiert werden muss:

1. Beschleunigung: Technische Innovationszyklen beschleunigen sich durch veränderte Produktionsbedingungen und durch die Logik unserer Märkte. Unsere Kommunikationspraktiken beschleunigen sich vor allem durch die digitalen Kommunikationstechniken. Dies führt zu einer Beschleunigung von gesellschaftlichen Praktiken und unserer Zeitwahrnehmung (Rosa 2016). Beschleunigte Innovationszyklen und Erkenntnisprozesse, führen zu einer geringeren Stabilität von verwendeter Technik und von gesellschaftlichen Praktiken sowie $\mathrm{zu}$ einer teils rasanten Modifikation von begründenden Erkenntnissen und Überzeugungen. Wenngleich das viel beschworene Mantra einer ubiquitär abnehmenden Halbwertzeit von Wissen mit Vorsicht zu genießen ist (Wolff 2008), wirkt sich dieser Beschleunigungsprozess auch auf die Persistenz der in einem Curriculum zusammengefassten Lerninhalte aus.

2. Interdependenz von Fachwissen und inter- und transdisziplinären Kompetenzen: Die Rekonstruktion jüngerer krisenhafter Ereignisse, wie zum Beispiel der Reaktorkatastrophe in Fukushima, oder langfristiger Herausforderungen, wie der Klimakrise, bestätigen immer wieder die Erkenntnis, dass Herausforderungen unserer globalisierten und digitalisierten Welt durch Interdependenzen zahlreicher Einflussgrößen sowie durch die Notwendigkeit einer mehrperspektivischen Betrachtungsweise als komplexe Probleme zu charakterisieren sind. 
Die Lösung komplexer Probleme erfordert das erfolgreiche inter- und transdisziplinäre Zusammenwirken von Spezialist*innen unterschiedlicher Disziplinen und Praxisfelder (Langemeyer 2015). Wie lässt sich diese Entwicklung einer stabilen fachlichen Bildung mit der Entwicklung überfachlicher Kompetenzen und kooperativer Mindsets optimal in Curricula abbilden? Hier ist die Integration von projektförmiger, praxis- und forschungsorientierter Lehre ein häufig genannter Lösungsweg (Speck und Schubart 2017). In der Realität der Curriculumentwicklung konkurriert der flexible Möglichkeitsraum, den derartige Lehr-Lern-Konzepte benötigen, jedoch häufig mit der Vorstellung, die Vermittlung standardisierbarer Wissens- und Kompetenzkataloge für die gesamte Studierendenkohorte realisieren zu können.

Diese Herausforderungen treffen auf eine oft geringe Flexibilität von Hochschulcurricula: Sobald Studierende in einem Studiengang immatrikuliert wurden, genießen sie (prüfungsrechtlichen) Vertrauensschutz. Das bedeutet, dass inhaltliche und didaktische Veränderungen, sowie sie Inhalt der Studien- und Prüfungsordnung sind, in vielen Fällen nicht kurzfristig und nur mit viel administrativem Aufwand möglich sind. Daraus folgt die wesentliche Herausforderung für den Curriculumentwicklungsprozess, wie Curricula flexibler gestaltet werden können, ohne grundlegende Ansprüche an die Vermittlung qualitativ hochwertiger Inhalte aufzugeben.

An einzelnen Standorten existieren bereits richtungsweisende Beispiele, wie dies in akkreditierten Studiengängen möglich gemacht werden kann:

- Über Studiengänge hinweg strukturell gemeinsame Experimentierräume im Curriculum schaffen: Zum Beispiel finden die interdisziplinären Wochen an der FH Kiel immer für zwei Wochen etwa zur Mitte der Vorlesungszeit statt. Die reguläre Lehre pausiert und wird durch ein fachbereichsübergreifendes Experimentierfenster für Lehrende und Studierende ersetzt. Alle Lehrenden können Veranstaltungen in unterschiedlichsten Formaten (zum Beispiel Workshops, Tagungen, Projekte) anbieten. Dabei werden vor allem interdisziplinäre innovative Themen und Formate adressiert. Die Studierenden können sich ein individuelles Programm für die 14 Tage zusammenzustellen (Sperga 2018).

- Ganzheitliche Lernerfahrungen durch projektförmige Lehre und forschendes Lernen: Zum Beispiel wurde an der FH Potsdam mit dem Konzept InterFlex eine Lehr-Lern-Form realisiert, bei der auf einen semesterweise erfolgenden Call zwei oder mehr Lehrende aus unterschiedlichen Disziplinen - auch Studierende können Lehrende sein - ein gemeinsames Projektseminar anbieten. 
Diese Seminare sind häufig thematisch angelehnt an Forschungs- oder Transferprojekte der beteiligten Lehrenden. In die Curricula der Studiengänge der Hochschule ist ein Flex-Fenster für die Teilnahme der Studierenden an diesen Veranstaltungen integriert (Ammann et al. 2013).

- Studiengang-, fachbereich- oder hochschulweites Semestermotto zur gezielten Auseinandersetzung mit aktuellen Themen: Zum Beispiel fördert seit 2017 der Stifterverband die hochschulweite Auseinandersetzung mit einem gemeinsamen aktuellen Thema mit dem Titel „Eine Uni - ein Buch“. Dabei sollen sich möglichst viele Mitglieder einer Universität über ein gemeinsames Thema oder Anliegen austauschen. Als Leitmotiv dient bei diesen Projekten jeweils ein Buch (von den Fabeln des Äsop bis zu Shaun Tans Graphic Novel „The Arrival“). Die Dokumentation der geförderten Projekte der letzten Jahre zeigt, mit welch unterschiedlichen Formaten dies gelingen kann (zum Beispiel Blogs, Podiumsdiskussionen, Lehrveranstaltungen, Fachvorträgen, Ausstellung) (Stifterverband für die Deutsche Wissenschaft o. D.a).

Für die Curriculumentwicklungspraxis empfehlen wir folgende Fragen auf Studiengangs- oder Fachbereichsebene zu diskutieren:

- Welche Rahmenbedingungen bietet unsere Hochschule bereits für die Integration inter- und transdisziplinärer Inhalte in unsere Curricula und wo müssen diese Rahmenbedingungen gegebenenfalls weiterentwickelt werden?

- Welche Lösungen haben wir bislang gefunden, um neue Themen schnell in unsere Curricula zu integrieren?

- Welche Strukturelemente können uns darüber hinaus helfen, Curricula flexibler zu gestalten?

- Wie regelmäßig müssen die Inhalte unserer Curricula (auch unabhängig von Akkreditierungszyklen) überprüft und angepasst werden (vgl. Seidl und Vonhof 2017)?

\section{$4 \quad$ Digital kompetente Lehrende}

Hochschulen und Lehrende müssen in die Lage versetzt werden, im Wandel zu bestehen und ihn gleichzeitig zu gestalten. Wie oben bereits gezeigt wurde, erfordert dies das Infragestellen und ggf. Verändern bewährter Prozesse und Organisationsmodelle. Dies gelingt aber nur, wenn die einzelnen Mitarbeitenden zum einen die Bereitschaft und Motivation, zum anderen aber auch das notwendige 
Know-how zur Gestaltung des Wandels mitbringen. Das heißt, es reicht nicht, den Studierenden das lebenslange Lernen zu predigen, sondern auch die Lehrenden müssen es für sich - individuell und als Organisation - als Herausforderung annehmen. Die Herausforderungen der Coronakrise zu Beginn der 2020er-Jahre haben eindrücklich gezeigt, wie herausfordernd dies ist.

Anknüpfend an das Modell der pädagogischen Hochschulentwicklung von Euler (vgl. Brahm 2016) können drei interdependente Handlungsebenen unterschieden werden, auf denen Maßnahmen zur digitalen Kompetenzentwicklung von Lehrenden angesiedelt sein können:

- „Ebene der Lernumgebungen: Kompetenzentwicklung der Lehrenden [...] damit verbunden - Gestaltung von Lehr-Lernumgebungen und Kurse.

- Ebene der Studienprogramme: Gestaltung von Profil und Zusammenwirken der Kurse in einem Studiengang.

- Ebene der Organisation: Gestaltung der strukturellen und kulturellen Rahmenbedingungen der Lehre an der Hochschule“ (Brahm 2016, S. 28).

Die individuelle Kompetenzentwicklung der Lehrenden: In diesem Bereich haben sich im Rahmen des Qualitätspakts Lehre vielfältige Formate und Organisationsformen entwickelt. Im Hinblick auf die digitale Kompetenzentwicklung der Lehrenden besteht eine doppelte Herausforderung: Zum einen muss die didaktische Kompetenz im digitalen Raum gefördert, zum anderen aber auch die Weiterentwicklung der Fachkompetenz der Lehrenden in den Blick genommen werden. Besonders Letzteres passiert heute weitgehend selbstständig und ungesteuert. Bei der didaktischen Weiterbildung der Lehrenden ist darauf Wert zu legen, dass das Lernen von digitalen Lehr-Lern-Methoden auch mithilfe von digitalen Lehr-Lern-Methoden erfolgt. Nur so kann das eigene Erleben der Lernendenperspektive in den Lernprozess integriert werden. Wie dies erfolgreich gelingen kann, zeigt beispielsweise der Kurs „Quickstarter Online-Lehre“ von e-teaching.org (o. D.).

Eine große Chance für das Lernen von- und miteinander im Hinblick auf die Entwicklung digitaler Kompetenzen liegt zudem auf der Ebene der Studienprogramme. Die Weiterentwicklung der Lehre auf der Ebene der Studiengänge kann stark vom Austausch, Peer-Lernen in Netzwerken und der Kooperation mit Partner*innen profitieren. Dies setzt Offenheit und Bereitschaft, aber auch passende Strukturen voraus. Dazu gehören etwa Orte, Zeit und Gelegenheit für Austausch, in die Studienstruktur eingebundene Formate für Kooperation und nicht zuletzt eine attraktive Berücksichtigung solcher Formate bei der Deputatsabrechnung. Formate des studiengangbezogenen Peer-Lernens der Lehrenden können 
(meta)reflexiv angelegt sein, zum Beispiel als Supervisionsgruppen oder „Experimental Learning Labs“ (vgl. Frankfurt University of Applied Sciences 2019), sie können Einblick in den jeweiligen Lehrprozess geben, wie zum Beispiel die an zahlreichen Hochschulen etablierten kollegialen Lehrhospitationen, oder sie können integriert in den Lehrprozess stattfinden, wie zum Beispiel das Team Teaching (vgl. Ammann et al. 2013). Neben der internen Kooperation sollten aber auch Formate für die Kooperation mit Praxispartner*innen im Curriculum Berücksichtigung finden. Diese erlauben den Lehrenden, Veränderungen im Praxisfeld zu erleben und dies als Lernmöglichkeiten für die Weiterentwicklung ihrer eigenen Fachkompetenz zu nutzen. Insbesondere im Austausch mit der Praxis und dem Vergleich der im eigenen Bildungsverlauf erlernten Praktiken mit der aktuellen Arbeitspraxis wird offenbar, wie eng Fachkompetenzen und Digitalkompetenzen zusammenhängen.

Auf der Ebene der Organisation stehen vor allem organisatorische, strategische und kulturelle Fragen im Mittelpunkt. Förderlich und hilfreich ist eine klare und geteilte Vorstellung der beteiligten Stakeholder über die digitalen Entwicklungsziele der Hochschule und daraus folgender Ziele für die digitale Kompetenzentwicklung der Lehrenden. Hier können Leitbildprozesse oder die Erarbeitung von Lehrverfassungen einen wichtigen Beitrag leisten (Wissenschaftsrat 2017). Wichtig ist jedoch auch der zweite Schritt, das heißt, Leitbilder mit Leben zu füllen und aus Visionen konkrete Ziele abzuleiten und umzusetzen. Dazu gehören etwa ein auf die Ziele ausgerichtetes Weiterbildungskonzept oder Maßnahmen zur Integration von verschiedenen Stakeholdern in Lehrentwicklungsprozesse im Sinne einer „Community of Teaching \& Learning“. Wie Peer-Lernen unter Lehrenden in Netzwerkstrukturen auch über Studiengangs- und Hochschulgrenzen hinweg organisiert und genutzt werden kann, zeigen etwa das Gutenberg Lehrkolleg an der Universität Mainz oder das Netzwerk Lehre ${ }^{\mathrm{N}}$. Herausragende Beispiele von Hochschulen, die über eine Lehrverfassung oder Lehrstrategie verfügen und Lehre auch als Experimentier- und Innovationsfeld begreifen, zeichnet der Stifterverband regelmäßig mit dem Genius Loci-Preis aus (Stifterverband für die Deutsche Wissenschaft o. D.b). Auf strategischer Ebene unterstützt das Hochschulforum Digitalisierung Hochschulen durch das Programm „Peer-to-PeerStrategieberatung zur Digitalisierung in Studium und Lehre“ bei der individuellen Weiterentwicklung. Die Dokumentation der geförderten Projekte zeigt, dass neben einer klaren Zielvorstellung die digitale Kompetenzentwicklung der Lehrenden an den beteiligten Hochschulen ein wesentlicher strategischer Entwicklungsbaustein ist (Hochschulforum Digitalisierung 2019). 
Die digitale Kompetenzentwicklung der Lehrenden ist kein Element der Curriculumentwicklung, aber sie ist eine Voraussetzung für die zukunftsorientierte Weiterentwicklung von Curricula. Daher empfehlen wir begleitend zur Curriculumentwicklungspraxis, die digitale Kompetenzentwicklung der Lehrenden anhand folgender Leitfragen in den Blick zu nehmen:

- Welche Herausforderungen stellt lebenslanges Lernen an die zukunftsorientierte Kompetenzentwicklung der Lehrenden eines Studiengangs und der gesamten Hochschule?

- Welche Angebote mit welchem Verpflichtungsgrad stehen zur Verfügung und sind diese angemessen?

- Wie und wo findet Peer-Lernen der Lehrenden statt?

- Welche ungenutzten Potenziale liegen in der Kooperation zwischen Lehrenden oder mit außerhochschulischen Partner*innen und wie können diese erschlossen werden?

- Welche Lehr-Lern-Kultur herrscht an der Hochschule und ist diese für die erwarteten Herausforderungen zielführend?

- Wie kann strategische und strukturelle Steuerung die zukunftsorientierte Kompetenzentwicklung der Lehrenden unterstützen?

\section{$5 \quad$ Fazit}

Die COVID-19-Pandemie hat der Gesellschaft und den Hochschulen eindrücklich den Entwicklungsbedarf im Hinblick auf das Lehren und Lernen im Zeitalter der Digitalisierung vor Augen geführt. Dabei geht es wie beschrieben zentral um die Frage, welche Kompetenzen heute und in Zukunft wichtig sind und wie beziehungsweise unter welchen Bedingungen sie sinnvoll in Curricula integriert werden können. Dabei muss jeder Studiengang - unter Einbeziehung der spezifischen Rahmenbedingungen und Stakeholder - den Stellenwert der Digitalisierung als Lerninhalt klären. Anschließend müssen Konzepte für flexible und den Kompetenzzielen angepasste Lehr-Lern-Formate entwickelt werden. Dies erfordert, die Kompetenz der Lehrenden und deren lebenslanges Lernen mit in den Blick zu nehmen. Bei der Curriculumentwicklung geht es um die Erarbeitung individuell passender Lösungen. Der eine richtige Weg existiert hier leider - oder zum Glück - nicht. Die Beispiele und Reflexionsfragen des Beitrags sollen jedoch dabei helfen, notwendige Denk- und Diskussionsprozesse an den Hochschulen zu gestalten. 
Netzwerke und Beratungsprogramme - wie etwa Lehre ${ }^{\mathrm{N}}$ im Bereich des individuellen Community Buildings oder der Peer-Beratung des HFD auf strategischer Ebene - führen bereits jetzt zu einer qualitativen Weiterentwicklung von Hochschulen und Hochschullehre. Für die Curriculumentwicklung und die 21st Century Skills besteht in diesem Bereich noch eine Lücke (vgl. dazu Seidl et al. o. D.) und dieser Beitrag kann vielleicht Impulse geben für die Realisierung eines Peer-Netzwerks zur Beratung bei der Weiterentwicklung von Curricula für das digitale Zeitalter. Die Jahrestagung der deutschen Gesellschaft für Hochschuldidaktik 2020 stand unter dem Motto „Hochschullehre als Gemeinschaftsaufgabe - miteinander, kooperativ, integrativ“. Leicht abgewandelt gilt dies auch für die hier behandelte Thematik: Curriculumentwicklung im Zeitalter der Digitalisierung ist eine wichtige Gemeinschaftsaufgabe, die nur miteinander, kooperativ und integrativ bewältigt werden kann.

\section{Literatur}

Akkreditierungsrat. (2013). Regeln für die Akkreditierung von Studiengängen und für die Systemakkreditierung. https://www.akkreditierungsrat.de/fileadmin/Seiteninhalte/ AR/Beschluesse/AR_Regeln_Studiengaenge_aktuell.pdf. Zugegriffen: 3. Juni 2020.

Ammann, B., Bartl, D., Cartes, S., \& Klose, B. (Hrsg.). (2013). InterFlex - Querdenken erwünscht: Interdisziplinär und forschend lernen. https://www.fh-potsdam.de/fileadmin/ user_upload/Lehren/FLEX/publikationen/interflex/interflex_broschuere_web.pdf. Zugegriffen: 3. Juni 2020.

Binkley, M., Erstad, O., Herman, J., Raizen, S., Ripley, M., Miller-Ricci, M., \& Rumble, M. (2012). Defining Twenty-First Century Skills. In P. Griffin \& E. Care (Hrsg.), Assessment and Teaching of 21st Century Skills. Methods and Approach (S. 17-66). Dordrecht: Springer.

Brahm, T. (2016). Pädagogische Hochschulentwicklung als Motor für die Qualitätsentwicklung von Studium und Lehre. In T. Brahm, T. Jenert, \& D. Euler (Hrsg.), Pädagogische Hochschulentwicklung. Von der Programmatik zur Implementierung (S. 19-36). Heidelberg: Springer.

Burmester, M., \& Seidl, T. (2020). Lehr-Lernkontexte in einer transformativen Fakultät. In R. Stang \& A. Becker (Hrsg.), Zukunft Lernwelt Hochschule (S. 86-98). Berlin: De Gruyter.

Digitale-Lehre-Germanistik. (2020). Digitale Konferenz. Während und nach Corona: Digitale Lehre in der Germanistik. https://vfr.mww-forschung.de/web/digitale-lehre-germanistik/ konferenz. Zugegriffen: 3. Juni 2020.

e-teaching.org. (o. D.). Quickstarter Online-Lehre. https://www.e-teaching.org/praxis/themen specials/quickstarter-online-lehre. Zugegriffen: 3. Juni 2020.

Floridi, L. (2014). The fourth revolution: How the infosphere is reshaping human reality. Oxford: Oxford University Press.

Frankfurt University of Applied Sciences. (2019). Peer Learning unter Kolleginnen und Kollegen. https://www.frankfurt-university.de/de/newsmodule/details/?tx_news_pi1\%5Bn 
ews $\% 5 \mathrm{D}=2681 \&$ tx_news_pi $\% 5 \mathrm{~B}$ controller\%5D=News\&tx_news_pi1\%5Baction\% 5D=detail\&cHash=e967da9e0a82a37b558356bca8210df5. Zugegriffen: 7. Juli 2020.

Fuchs, M. (2020). Digitales Sommersemester. Im Rausch der Online-Lehre. FAZ online, (8. Mai). https://www.faz.net/aktuell/karriere-hochschule/hoersaal/digitales-sommersem ester-im-rausch-der-online-lehre-16754543.html. Zugegriffen: 3. Juni 2020.

Gerholz, K.-H., \& Sloane, P. (2013). Studiengang- und Modulentwicklung - Aktuelle Herausforderungen und Potentiale zur forschungsorientierten Gestaltung. In K.-H. Gerholz \& P. Sloane (Hrsg.), Studiengänge entwickeln-Module gestalten. Eine Standortbestimmung nach Bologna (S. 5-28). Paderborn: Eusl.

Hochschul-Hackathon Brandenburg. (o. D.). Hochschul-Hackathon Brandenburg. https://hoc hschul-hackathon-bb.de/. Zugegriffen: 3. Juni 2020.

Hochschulforum Digitalisierung. (2018). Curriculumentwicklung für das digitale Zeitalter. Thesen und Empfehlungen der AG Curriculum 4.0 des Hochschulforum Digitalisierung (Arbeitspapier, 39). https://doi.org/10.5281/zenodo.2602541.

Hochschulforum Digitalisierung. (2019). Dimensionen und Handlungsfelder für die Hochschulbildung im digitalen Zeitalter. https://hochschulforumdigitalisierung.de/de/strategis che-handlungsfelder. Zugegriffen: 2. Juli 2020.

Hochschulforum Digitalisierung. (2020). Rückblick: \#SemesterHack - Wir hacken das digitale Sommersemester! https://hochschulforumdigitalisierung.de/de/online-hac kathon. Zugegriffen: 3. Juni 2020.

HRK Projekt Nexus. (2017). Studiengangentwicklung - von der Idee zum Curriculum. Nexus Impulse für die Praxis, 13. https://www.hrk-nexus.de/fileadmin/redaktion/hrknexus/07-Downloads/07-02-Publikationen/impuls_Nr.13_mit_Links.pdf. Zugegriffen: 3. Juni 2020.

Kirchherr, J., Klier, J., Lehmann-Brauns, C., \& Winde, M. (2019). Future Skills: Welche Kompetenzen in Deutschland fehlen. Future Skills - Diskussionspapier, 1. Essen: Stifterverband für die Deutsche Wissenschaft e. V. https://www.stifterverband.org/download/ file/fid/6360. Zugegriffen: 3. Juni 2020.

Langemeyer, I. (2015). Das Wissen der Achtsamkeit: Kooperative Kompetenz in komplexen Arbeitsprozessen. Münster: Waxmann.

Langen, F., \& Bosch, H. (2014). Massive open online courses: Disruptive innovations or disturbing inventions? Open Learning: The Journal of Open, Distance and e-Learning, 28, 216-226.

Michel, A., Langer, C., \& Heidmann, F. (2018). Curriculum design: How can the development of information science curricula benefit from interdisciplinary cooperation? In $\mathrm{T}$. Aparac-Jelusic, V. Casarosa, \& E. Maceviciute (Hrsg.), The future of education in information science. In Proceedings from FEIS - International EINFOSE Symposium. Osijek: University of Osijek. https://einfose.ffos.hr/feis-2018/proceedings. Zugegriffen: 3. Juni 2020.

Michel, A., Langer, C., \& Heidmann, F. (2019). Design 0815 - Curriculumentwicklung in den Design-Disziplinen. DUZ - Wissenschaft und Management, 2(10), 17-20.

Rosa, H. (2016). Beschleunigung: Die Veränderung der Zeitstrukturen in der Moderne (11. Aufl.). Frankfurt a. M: Suhrkamp.

Schaper, N. (2012). Fachgutachten zur Kompetenzorientierung in Studium und Lehre. https:// www.hrk-nexus.de/fileadmin/redaktion/hrk-nexus/07-Downloads/07-02-Publikationen/ fachgutachten_kompetenzorientierung.pdf. Zugegriffen: 3. Juni 2020. 
Seidl, T. (2017). Schlüsselkompetenzen als Zukunftskompetenzen. Die Bedeutung der „21st Century Skills“ für die Studiengangsentwicklung. In B. Berendt, B. Szczyrba, A. Fleischmann, N. Schaper, \& J. Wildt (Hrsg.), Neues Handbuch Hochschullehre (J 2.23, S. 89-114). Berlin: DUZ.

Seidl, T., Baumgartner, P., Brei, C., Gerdes, A., Lohse, A., Kuhn, S., Michel, A., Pohlenz, P., Quade, S., \& Spinath, B. (2018). (Wert-)Haltung als wichtiger Bestandteil der Entwicklung von 21st century skills an Hochschulen. Diskussionspapier, 3. Berlin: Hochschulforum Digitalisierung. https://doi.org/10.5281/zenodo.2634975.

Seidl, T., Michel, A., \& Kuhn, S. (o. D.). Future Skills brauchen Unterstützer an den Hochschulen. https://www.future-skills.net/meinungen/seidl-future-skills-brauchen-unterstue tzer-an-den-hochschulen. Zugegriffen: 3. Juni 2020.

Seidl, T., \& Vonhof, C. (2017). Agile Prinzipien - was kann die Studiengangsentwicklung davon lernen? Synergie, 3, 22-25.

Speck, K., \& Schubart, W. (2017). Perspektiven für Wirtschaft und Gesellschaft? Die Frage der Beschäftigungsfähigkeit und die Umsetzung des Forschenden Lernens. In H. A. Mieg \& J. Lehmann (Hrsg.), Forschendes Lernen: Wie die Lehre in Universität und Fachhochschule erneuert werden kann (S. 427-438). Frankfurt: Campus.

Sperga, M. (2018). Interdisziplinäre Wochen an der Fachhochschule Kiel. Aktuelles Konzept und Erfahrungen. In U. Beer, C, Metzger, \& A. Rieck (Hrsg.), Lehre und Lehrentwicklung an Fachhochschulen (S. 142-246). Münster: Waxmann.

Stifterverband für die Deutsche Wissenschaft. (o. D.a). Eine Uni - ein Buch. https://www.sti fterverband.org/eine-uni-ein-buch. Zugegriffen: 3. Juni 2020.

Stifterverband für die Deutsche Wissenschaft. (o. D.b). Genius Loci-Preis für Lehrexzellenz. https://www.stifterverband.org/genius-loci. Zugegriffen: 3. Juni 2020.

TH Köln. (o. D.). Curriculumswerkstatt. https://www.th-koeln.de/hochschule/studiengangs-und-curriculumsentwicklung_49326.php. Zugegriffen: 3. Juni 2020.

Venn, M. (2013). Studiengänge gemeinsam verbessern - Der Student's Day als partizipatorisches Instrument der Evaluation und Studiengangsentwicklung. die Hochschullehre. https://www.hochschullehre.org/?dl_id=41. Zugegriffen: 3. Juni 2020.

Wissenschaftsrat. (2017). Strategien für die Hochschullehre. Positionspapier. https://www. wissenschaftsrat.de/download/archiv/6190-17.pdf;jsessionid=E0244867FE2BD29689 49DB668F0B8B96.delivery 1-master?_blob=publicationFile\&v=3. Zugegriffen: 3 . Juni 2020.

Wolff, C. (2008). Die Halbwertszeit der Wissenszwerge. Anmerkungen zu einigen „Mythen“ der Wissensgesellschaft. In A. Geisenhanslüke \& H. Rott (Hrsg.), Ignoranz (S. 203-228). Bielefeld: transcript.

World Economic Forum. (2016). The future of jobs. Employment, skills and workforce strategy for the fourth industrial revolution. https://www3.weforum.org/docs/WEF_Future_ of_Jobs.pdf. Zugegriffen: 3. Juni 2020. 
Open Access Dieses Kapitel wird unter der Creative Commons Namensnennung 4.0 International Lizenz (http://creativecommons.org/licenses/by/4.0/deed.de) veröffentlicht, welche die Nutzung, Vervielfältigung, Bearbeitung, Verbreitung und Wiedergabe in jeglichem Medium und Format erlaubt, sofern Sie den/die ursprünglichen Autor(en) und die Quelle ordnungsgemäß nennen, einen Link zur Creative Commons Lizenz beifügen und angeben, ob Änderungen vorgenommen wurden.

Die in diesem Kapitel enthaltenen Bilder und sonstiges Drittmaterial unterliegen ebenfalls der genannten Creative Commons Lizenz, sofern sich aus der Abbildungslegende nichts anderes ergibt. Sofern das betreffende Material nicht unter der genannten Creative Commons Lizenz steht und die betreffende Handlung nicht nach gesetzlichen Vorschriften erlaubt ist, ist für die oben aufgeführten Weiterverwendungen des Materials die Einwilligung des jeweiligen Rechteinhabers einzuholen.

(c) (P) 